\title{
Formally speaking
}

\section{Mathematical theorems can be created by formalization of everyday expressions.}

\section{John L. Casti}

$\mathrm{t}$ is often said that words can kill. But they can also create. Mathematics offers an excellent example of how this creative process works. Consider, for example, the common phrase, "Beauty is in the eye of the beholder". The utility of this phrase for everyday life resides in the ambiguous term 'beauty'. What does it really mean? Well, no one can say objectively, as the term is used in daily speech to mean a plethora of often contradictory things; 'beauty' is an informal notion, and therein lies its beauty (if you'll pardon the pun). But mathematicians hate the informal; it is hard to prove theorems without having a clear-cut notion of what the objects of your theory actually mean. So a lot of mathematics has been developed around just this idea: formalization of the informal.

A good illustration of this formalization process is Kurt Gödel's famous result on the incompleteness of logical systems. This result revolves about the notion of the truth of propositions about numbers. Is it 'true' that $1+1=2$ ? The logician Alfred Tarski showed in the 1920s that it is simply not possible to capture completely the everyday concept of 'truth' within the confines of a fixed logical system.

A large part of Gödel's genius was seeing how to replace the informal idea of truth with a concept that can be formalized, the idea of proof. Using the formal notion of proof, Gödel then showed that there must exist some statement about numbers that cannot be proved or disproved using the rules of logical deduction - but that the statement can actually be seen to be true by jumping outside the logical system and viewing the statement from a metalinguistic perspective.

There are many ways to state this result in linguistic terms. My favourite is to say

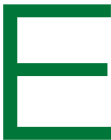

veryday language is incredibly rich in its ability to evoke images and intuitive concepts, but to prove theorems it must be somewhat constrained. that there is something fishy that one must always remember that number theory is about numbers. In other words, Gödel's incompleteness theorem shows that there is an irreducible semantic component to mathematics. It is not just a game of shuffling meaningless strings of symbols about in accordance with a set of transformation rules. The objects of mathematics really do mean something, and that meaning cannot be completely replaced by pure syntax alone.

Here is another example of how formalization of the informal leads to new mathematics. Consider the seemingly innocent query: "What is the smallest number that cannot be expressed in words?" This statement seems to pick out a definite number. Let's call it $U$ for 'unnameable'. But a moment's thought shows about this labelling. On the one hand, we seem to have just described the number $U$ in words. But $U$ is supposed to be the first number that cannot be described in words! A variation of this paradox seems to have originally been suggested to Bertrand Russell by a certain Mr G. G. Berry, a librarian at Oxford University.

The Berry paradox contains its own unformalizable notion, the concept of denotation between the terms in its statement and numbers. In arriving at his results on randomness, complexity and the limitations of rule-based knowledge, Gregory Chaitin of the IBM Watson Research Center had the insight to see that the way to formalize this concept was to shift attention to the phrase: "the smallest number not computable by a program of complexity $N$ ". This phrase can be formalized by specifying a certain computer program for searching out such a number. What Chaitin discovered was that no program of complexity $N$ can ever produce a number having complexity greater than $N$. Therefore, the program of complexity $N$ can never halt by outputting the number specified by Chaitin's phrase. This fact constitutes an algorithmic complexity version of the unsolvability of the halting problem, a computer science version of Gödel's incompleteness theorem.

In fact, the halting problem itself is the end result of a formalization process by Alan

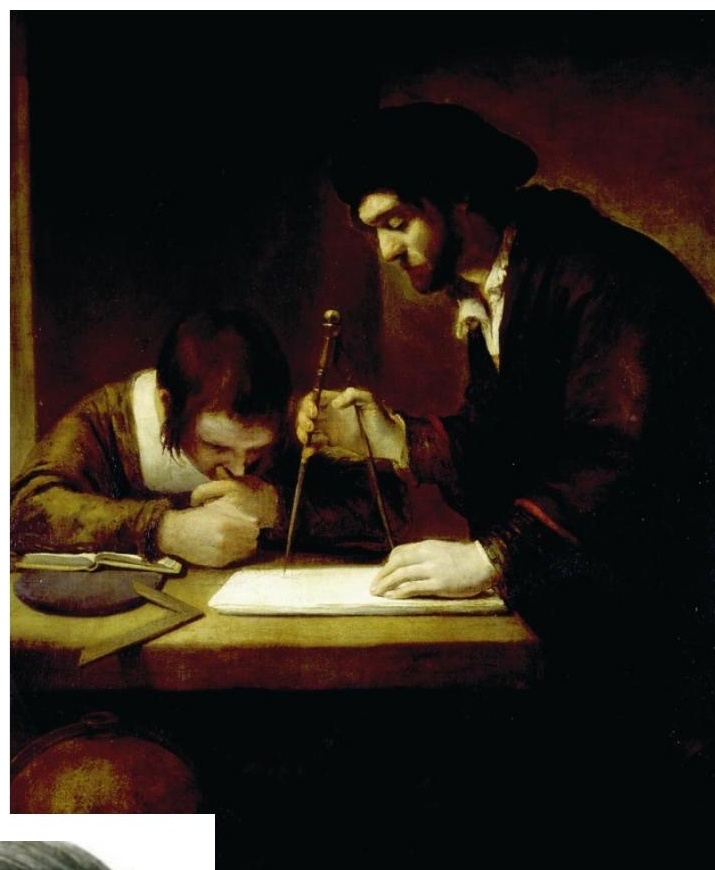

New ideas: traditional mathematics has advanced by virtue of formalizations such as Alan Turing's (left) abstract computer.

Turing, who in the mid1930s considered the informal idea of a "computation". Turing's way of formalizing this notion was to create an abstract computer now called a Turing machine, whose structure provides the basis for the zillions of computers in use every day around the world. If this doesn't show the power of words to create, nothing does!

The history of mathematics is filled with other examples of this sort, each formalization leading to the development of a major branch of mathematics. We can mention the notions of 'rational' decision-making and game theory, 'closeness' and topology, and 'infinity' and calculus, as illustrations of this claim. In a more contemporary vein, one can only wonder when the equally informal idea of 'complex' will be formalized into a rich mathematical theory of complex systems.

In all cases, what is involved in this process is the realization that everyday language is incredibly rich in its ability to evoke images and intuitive concepts. But to prove theorems it is necessary to constrain this expressive power somewhat, while at the same time not suffocating it entirely. Finding the right formalization is a large component of the art of doing great mathematics.

John Casti is at the Institute of Econometrics, Operational Res \& System Theory, Technical University of Viennna, Argentinierstrasse 8, A-1040 Vienna, Austria. 